\title{
Colistin dampens fibrinolysis and endothelial activation during endotoxaemia
}

\section{A randomised, double blind trial}

\author{
Christian Schoergenhofer ${ }^{1}$; Peter Matzneller'; Marion Mußbacher2; Johannes A. Schmid²; Petra Jilma-Stohlawetz ${ }^{3}$; \\ Markus Zeitlinger ${ }^{1} ;$ Bernd Jilma $^{1}$ \\ 'Department of Clinical Pharmacology, Medical University of Vienna, Vienna, Austria; ${ }^{2}$ Department of Vascular Biology and Thrombosis Research, Center for Physiology and \\ Pharmacology, Medical University of Vienna, Vienna, Austria; ${ }^{3}$ Department of Laboratory Medicine, all at Medical University of Vienna, Vienna, Austria
}

\begin{abstract}
Summary
Colistin electrostatically interacts with lipopolysaccharides (LPS). Preclinical studies demonstrated beneficial effects of colistin on LPS-induced coagulation and fibrinolysis. The objective of this trial was to investigate the effects of colistin during experimental endotoxaemia. In this randomised, double-blind, placebo-controlled, crossover trial 16 healthy volunteers received a $2 \mathrm{ng} / \mathrm{kg}$ LPS bolus after infusion of 2.5 million IU colistin or placebo. Plasma levels of F1+2 prothrombin fragments, thrombin-antithrombin complexes (TAT), von Willebrand factor antigen levels (vWF), E-selectin, plasmin-antiplasmin complexes (PAP), tissue-type plasminogen activator (t-PA) antigen and activity, plasminogen activator inhibitor-1 (PAI-1) were measured. Infusion of colistin significantly reduced peak concentrations of PAP complexes
\end{abstract}

\section{Correspondence to:}

Bernd Jilma, MD

Department of Clinical Pharmacology

Medical University of Vienna

Währinger Gürtel 18-20, 1090 Vienna, Austria

Tel.: +43140400 29810, Fax: +4314040029980

E-mail: bernd.jilma@meduniwien.ac.at

Note: This work was conducted at the Medical University of Vienna. EudraCT-Nr.: 2014-00285720

Supplementary Material to this article is available online at www.thrombosis-online.com. by $70 \%$, t-PA antigen levels by $63 \%$ and t-PA activity by $48 \%$, while PAI-1 levels decreased numerically by $63 \%$. Two hours after the LPS bolus F1+2 levels and TAT complexes were slightly reduced in the colistin period, but peak concentrations were similar in both periods. Colistin blunted the LPS induced four-fold increase in soluble E-Selectin levels by $\sim 50 \%$ and the two-fold increase in vWF antigen levels by $\sim 70 \%$. The LPS-scavenging actions of colistin significantly reduce endothelial activation and fibrinolytic response in the human endotoxaemia model, while the activation of the coagulation system remains largely unaffected.

\section{Keywords}

Coagulation, colistin, endotoxaemia, fibrinolysis, endothelial cells

Financial support:

This work was supported by the Austrian Science Funds (FWF) with the grant number SFB54P04.

Received: March 20, 2017

Accepted after major revision: May 20, 2017

Epub ahead of print: August 10, 2017

https://doi.org/10.1160/TH17-03-0196

Thromb Haemost 2017; 117: 1714-1721

\section{Introduction}

Colistin was introduced to clinical use in the 1950s (1). It's an effective antimicrobial agent against gram-negative and mycobacterial species. Due to its neuro- and nephrotoxicity colistin was replaced by other less toxic antimicrobial substances in the following decades $(1,2)$. The interest in colistin reemerged with the increase in multi-drug resistant gram-negative bacteria in recent years, most prominently Pseudomonas aeruguinosa, Acinetobacter baumannii and Klebsiella pneumonia $(1,3)$. Furthermore colistin may be used in combination with other antimicrobial substances to break up biofilms (4). Colistin exerts its bactericidal effects by interacting electrostatically with the anionic portion of lipopolysaccharides (LPS), which is located at the outer membrane of gram-negative bacteria (5). It competitively displaces divalent cations $\mathrm{Ca}^{2+}$ and $\mathrm{Mg}^{2+}$ bridges that stabilise LPS and thereby disintegrates the cellu- lar membrane (6). This subsequently causes leakage of cellular contents and eventually bacterial death (7).

Colistin may also exert anti-inflammatory effects by binding and neutralising LPS (8). This was shown in various animal models with promising results (8-10), i.e. colistin reduced TNF- $\alpha$, IL-1 $\beta$ and IL-6 levels and improved survival in a pneumonia mouse model (8). Furthermore, infusion of colistin reduced the inflammatory response in a human endotoxaemia model (11).

However, endotoxaemia does not only cause an inflammatory response but an intertwined, tissue-factor driven activation of the coagulation system (12). This coagulatory response is counterbalanced by a release of components of the fibrinolytic system demonstrated by increased levels of tissue-type plasminogen activator (t-PA), plasminogen activator inhibitor-1 (PAI-1), plasmin-antiplasmin complexes (PAP) and D-dimers (13). Indeed, colistin prevented the endotoxin-induced disseminated intravascular coagu- 
lopathy in a rabbit model (10). Furthermore colistin has prevented the LPS-induced production of inhibitors of fibrinolysis in vitro (14).

The endothelium plays a crucial role in the interplay between coagulation, inflammation and fibrinolysis and markers of endothelial activation markedly increased after infusion of LPS (15). This endothelial activation is considered to be largely depending on TNF- $\alpha$, as blockade of TNF- $\alpha$ almost entirely blunted the LPSinduced increase in E-selectin (16).

Based on these pre-clinical studies we hypothesised that colistin may have beneficial effects on coagulation activation, the fibrinolytic response and endothelial activation during experimental endotoxaemia. Experimental endotoxaemia is a standard model of acute inflammation in healthy volunteers $(17,18)$. Therefore in this randomized, placebo-controlled trial we investigated the effects of colistin on the LPS induced activation of coagulation and fibrinolysis in experimental endotoxaemia in healthy volunteers.

\section{Materials and methods}

The trial was conducted at the Department of Clinical Pharmacology, at the Medical University of Vienna between September and December 2014 and was performed in accordance with the Good Clinical Practice guideline and the principles set forth in the Declaration of Helsinki. The independent ethics committee of the Medical University of Vienna and the national authority (AGES, Austrian Agency for Health and Food Safety) gave their approval before the trial was initiated. The trial was registered at the $\mathrm{Eu}-$ draCT database with the identifier 2014-00285720. Written and oral informed consent to participate in the trial was obtained from all healthy volunteers before any trial-related activity was performed.

\section{Healthy volunteers}

Sixteen male, healthy volunteers aged 19-40 years were included in this trial. Major inclusion criteria comprised normal laboratory parameters, normal medical history and normal findings in the physical examination during the screening visit. Key exclusion criteria were allergies or hypersensitivities to any of the used products, a history of anaphylaxis or severe allergic reactions, coagulopathies or known coagulation disorders, recent participation in trials with investigational drugs or LPS, a body weight $<60$ or $>95$ $\mathrm{kg}$, intake of any medication within one week of the first trial day, a clinically relevant illness within three weeks prior to the trial days and liver or kidney dysfunction.

\section{Study design}

This was a prospective, investigator- and participant-blinded, single-center, randomised, two-way crossover trial. Subjects were randomly allocated to receive an infusion of 2.5 million IU of colistin or $250 \mathrm{ml} 0.9 \%$ saline solution as a placebo over 60 minutes (min) followed by a bolus infusion of $2 \mathrm{ng} / \mathrm{kg}$ bodyweight LPS (US
Standard Reference Endotoxin E. coli, CC-RE- Lot 2) over 1-2 $\mathrm{min}$. Infusion of placebo or colistin was initiated $50 \mathrm{~min}$ before infusion of LPS. This dosing schedule was chosen to allow both drugs to reach their maximum effect simultaneously, approximately 1.5 hours $(\mathrm{h})$ after infusion of the LPS bolus $(11,19)$. After a washout period of 6 weeks subjects received the alternate treatment in a crossover manner.

\section{Trial day}

On the trial days subjects reported to the ward in the morning after an overnight fast. Following the infusion of LPS all subjects received a continuous infusion of $100 \mathrm{ml} / \mathrm{h} 0.9 \%$ saline solution for a duration of $8 \mathrm{~h}$. Vital signs, including body temperature, heart rate and blood pressure, were monitored. To alleviate potential LPS-triggered flu-like symptoms paracetamol $(500 \mathrm{mg}$ tablet or $1000 \mathrm{mg}$ infusion) was available for all subjects (20). Subjects received a standardised meal $4 \mathrm{~h}$ after infusion of LPS. At $8 \mathrm{~h}$ after infusion of LPS, subjects were discharged from the study ward. The next morning, $24 \mathrm{~h}$ after the LPS bolus another visit was planned.

\section{Laboratory analysis}

Blood sampling was performed at pre-defined time-points: at the baseline, as well as $1 \mathrm{~h}, 2 \mathrm{~h}, 4 \mathrm{~h}, 6 \mathrm{~h}, 8 \mathrm{~h}$ and $24 \mathrm{~h}$ after the infusion of LPS. Differential blood counts were performed by the accredited central laboratory of the General Hospital of Vienna using the Sysmex XE-2100 / XE-5000 as previously described. To determine the effects of colistin on the activation of coagulation and fibrinolysis in the endotoxaemia model we performed commercially available enzyme linked immunoassays (ELISA) to measure levels of prothrombin fragments F1+2 (F1+2) (Enzygnost ${ }^{\circ} \mathrm{F} 1+2$, Siemens), thrombin-antithrombin complexes (TAT) (Enzygnost ${ }^{\bullet}$ TAT, Siemens), plasmin-antiplasmin complexes (PAP) (PAP Elisa, $\mathrm{DRG}^{\bullet}$ International Inc.), plasminogen activator inhibitor-1 (PAI-1) (Technozym ${ }^{\bullet}$ PAI-1 Actibind ${ }^{\oplus}$, Technoclone), tissue-type plasminogen activator (t-PA) (Technozym ${ }^{\circledR}$ t-PA Combi Actibind ${ }^{\circledR}$ ) antigen (Ag) and activity (Act). To investigate the influence of colistin on endothelial activation during endotoxaemia we measured soluble E-selectin by ELISA (sE-Selectin/CD62E Quantikine, R\&D Systems) and von Willebrand Factor (vWF) antigen levels (STA LIA test, Diagnostica Stago) (22). All assays were performed according to the manufacturers' instructions.

\section{Endpoints}

The main endpoint of this trial were plasma levels of prothrombin fragments F1+2. Secondary endpoints included platelet counts, TAT levels, t-PA antigen levels and activity, E-selectin levels, vWF antigen levels, PAP complex levels and PAI-1 levels. Safety assessments included vital parameters and documentation of adverse events and concomitant medication. 


\section{Randomisation and blinding}

Block randomisation with block sizes of 8 was performed using an open access randomisation generator (www.randomisation.com). An unblinded study nurse under supervision of an unblinded physician who had access to treatment allocation codes prepared study drugs. These were not otherwise involved in the conduct of the trial. Participants, investigators and laboratory staff were blinded. Both study drugs were not distinguishable from each other by color or physical properties.

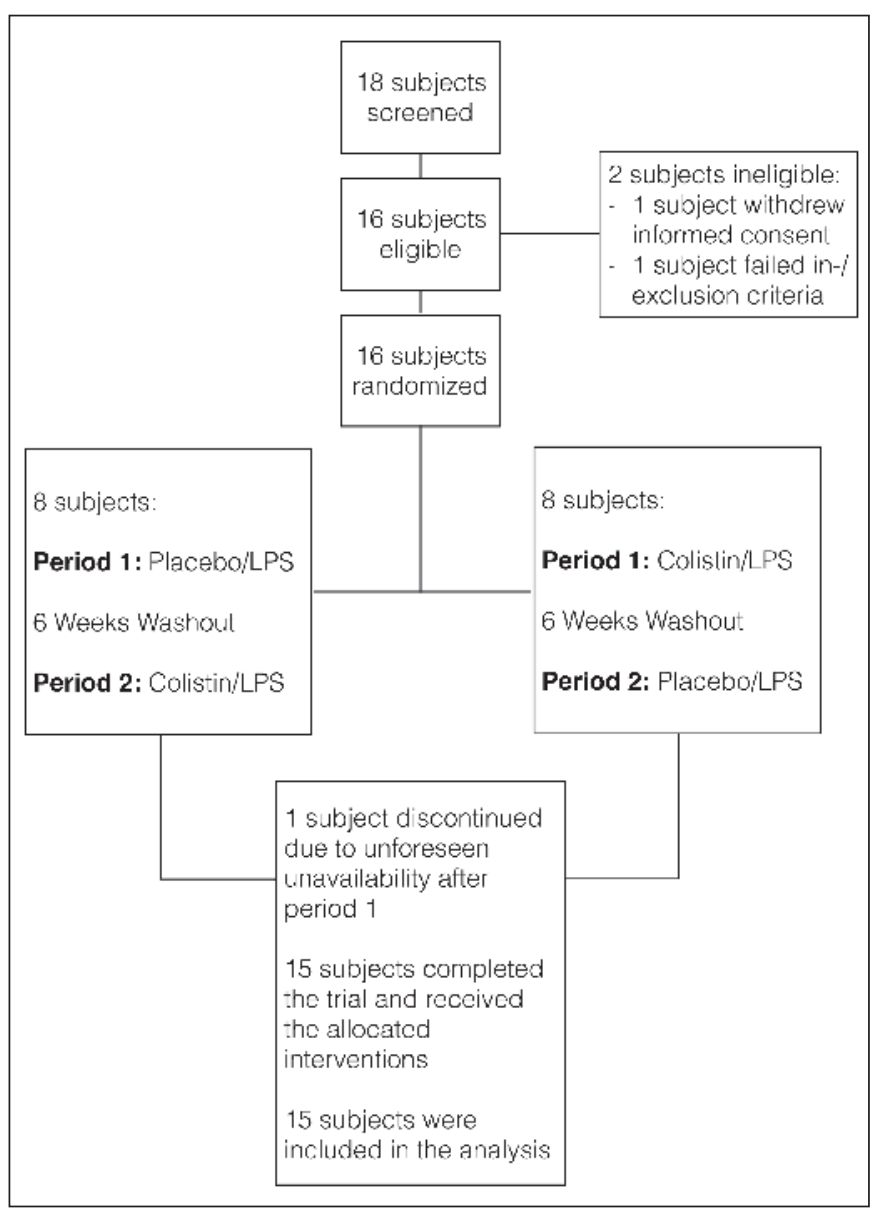

Figure 1: Flow chart.

Table 1: Baseline data and demographics.

\begin{tabular}{|l|l|}
\hline Parameter & Means $\pm 95 \%$ Confidence Intervals \\
\hline Age [years] & $28 \pm 2$ \\
\hline Male $[\mathrm{n}]$ & 16 \\
\hline Height $[\mathrm{cm}]$ & $184 \pm 3$ \\
\hline Weight $[\mathrm{kg}]$ & $79 \pm 3$ \\
\hline Platelets $\left[{ }^{*} 10^{9} / \mathrm{l}\right]$ & $215 \pm 42$ \\
\hline
\end{tabular}

\section{Sample size}

The sample size calculation was based on the results of previous trials involving anticoagulants in the human endotoxaemia model, where 10 to 15 subjects were sufficient to detect significant differences in the main outcome parameter $(\mathrm{F} 1+2$ levels) between treatment groups in parallel-group studies $(23,24)$. No data was available on the effects of colistin on prothrombin fragments F1+2. A peak $\mathrm{F} 1+2$ concentration of $819 \pm 173 \mathrm{pmol} / \mathrm{ml}$ was measured in a previous trial (25). Assuming a $25 \%$ reduction in this increase a sample size of 12 would suffice to detect a significant difference with a power of $80 \%$ and p-value of 0.05 . By increasing the sample size to 15 subjects a power of $90 \%$ could be reached. Therefore, and to account for potential drop-outs, a sample size of 16 healthy volunteers was chosen.

\section{Statistics}

All data are expressed as mean \pm SD or $95 \%$ confidence intervals (CI) unless otherwise stated. A repeated measures ANOVA was used for analysis of treatment and period effects on outcome parameters. If significant, (pair wise) post-hoc analysis using nonparametric tests for reasons of robustness were performed, i.e. the Friedman ANOVA and the Wilcoxon test, as appropriate. Eight parameters were tested in total and the p-value of 0.05 was corrected for multiple testing. A two-sided p-value of 0.00625 was considered significant. All statistical evaluations were performed using commercially available statistical software (IBM, SPSS 22).

If measured levels were below the detection limit of the respective assay, the detection limit was imputed (e.g. $2 \mathrm{IU} / \mathrm{ml}$ for t-PA activity).

\section{Results}

Between September 23 and September 29, 201416 healthy volunteers were screened for participation in this trial. The trial was completed after the last follow-up visit on December $9^{\text {th }} 2014$. Fifteen healthy volunteers completed the study ( $\$$ Figure 1 ). Baseline data and demographics are presented in Table 1. One subject missed study period II due to unforeseen unavailability and was excluded from analysis. The anti-inflammatory effects of colistin in the human endotoxaemia model were published recently (26); in essence colistin reduced TNF- $\alpha$, IL-6 and IL-8.

\section{Coagulation}

Infusion of $2 \mathrm{ng} / \mathrm{kg}$ LPS increased F1+2 levels several-fold in both periods with slightly higher levels in the placebo period. Overall there was no difference between study periods, although plasma levels of $\mathrm{F} 1+2$ seemed to be lower in the colistin group $2 \mathrm{~h}$ after infusion of LPS, as confidence intervals did not overlap with means of the other period ( $>$ Figure 2).

Plasma levels of TAT increased in both study periods severalfold with similar peak concentrations. There was no difference be- 
Figure 2: Coagulation specific biomarkers after infusion of an LPS bolus ( $2 \mathrm{ng} / \mathrm{kg}$ ) and infusion of colistin or placebo. Presented are means $\pm 95 \%$ confidence intervals $(\mathrm{Cl})$. Prothrombin complexes $\mathrm{F} 1+2(\mathrm{~A})$ and ThrombinAntithrombin (TAT) levels (B) are presented. No significant differences between both periods were found. $(n=15)$.

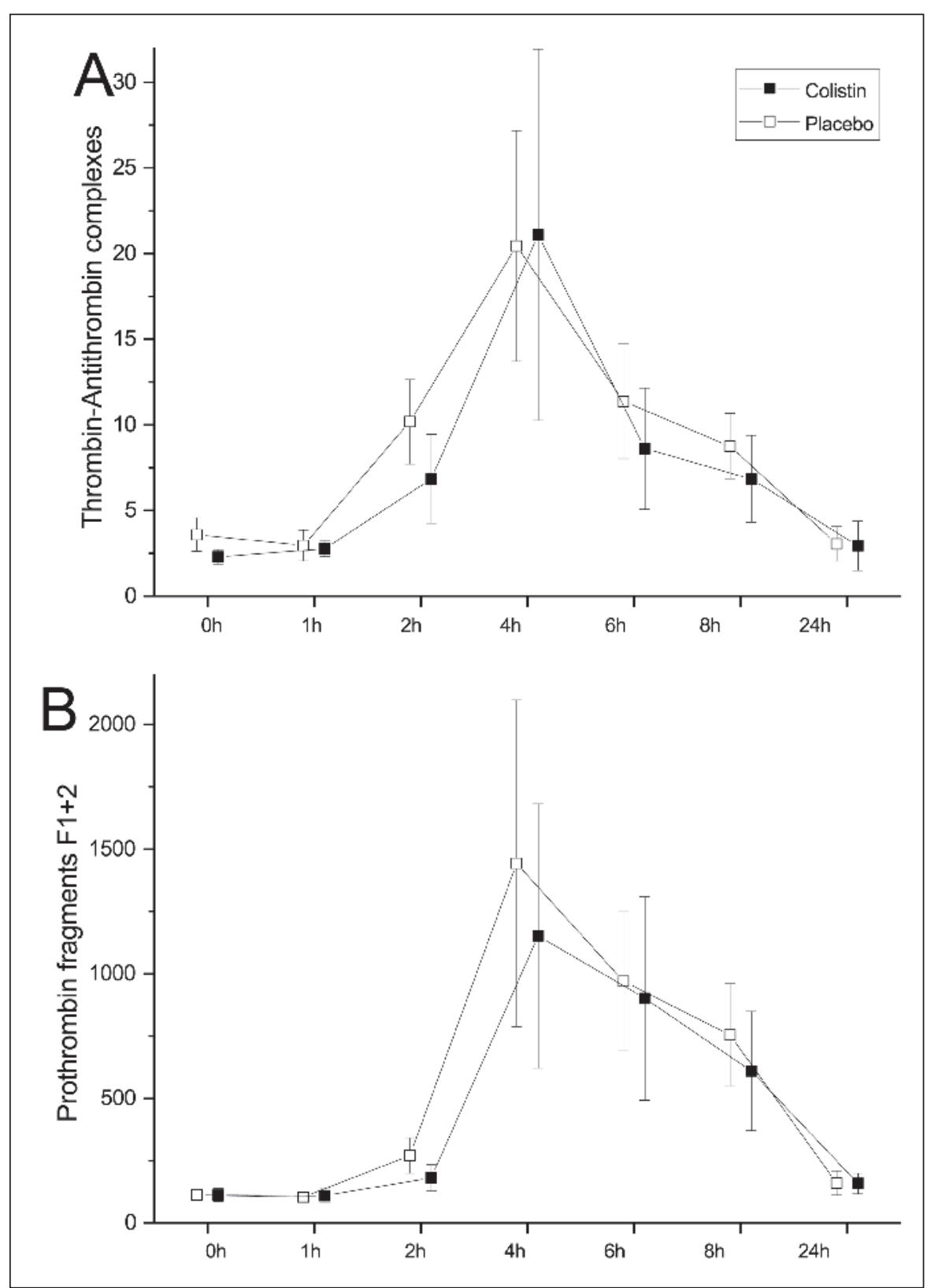

tween both study periods. In the colistin period, TAT levels were lower $2 \mathrm{~h}$ after infusion of LPS as indicated by the confidence intervals that did not overlap with means of the other period ( $>$ Figure 2).

Platelet counts transiently decreased by approximately $20 \% 6 \mathrm{~h}$ after the LPS bolus in both study periods. However, there was no significant difference between colistin and placebo (Suppl. Figure 1 , available online at www.thrombosis-online.com). Platelet counts normalised after $24 \mathrm{~h}$.

\section{Fibrinolysis}

Infusion of LPS increased PAP levels 4.7-fold in the placebo period, but only 1.7 -fold in the colistin period. Colistin significantly lowered PAP complex levels ( $\mathrm{p}<0.001,>$ Figure 3 ) after infusion of LPS. The peak PAP concentration was approximately $70 \%$ lower in the colistin period compared to placebo.

Colistin significantly reduced t-PA antigen levels after infusion of the LPS bolus ( $\mathrm{p}<0.001,>$ Figure 3 ). In the placebo period the peak concentration of t-PA activator antigen was $25.4 \pm 14.9 \mathrm{ng} / \mathrm{ml}$ (mean $\pm \mathrm{SD}$ ) $2 \mathrm{~h}$ after the LPS bolus. This increase was almost completely blunted by colistin and the peak concentration was 7.1 $\pm 1.8 \mathrm{ng} / \mathrm{ml}$ (mean $\pm \mathrm{SD}$ ). In both study periods, tissue-type plasminogen activator activity increased $2 \mathrm{~h}$ after infusion of LPS. No significant differences were detected between study periods (placebo: $4.0 \pm 3.1 \mathrm{IU} / \mathrm{ml}$ vs colistin: $6.9 \pm 6.6 \mathrm{IU} / \mathrm{ml}$ ) (data not shown).

Infusion of LPS increased PAI-1 levels $\sim 20$-fold in the placebo period and $\sim 6$-fold in the colistin period after $4 \mathrm{~h}$. In the colistin 


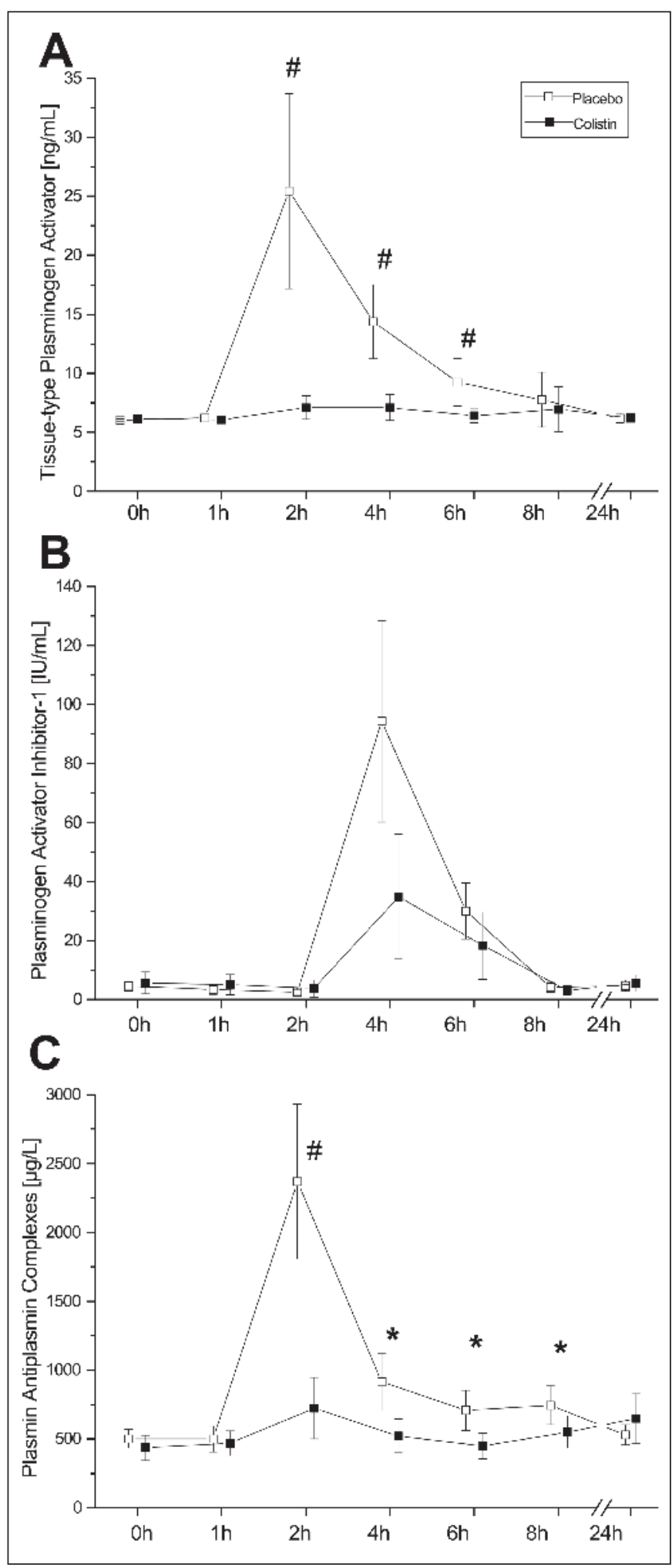

Figure 3: Fibrinolysis specific parameters tissue-type plasminogen activator (t-PA) antigen (A), plasminogen activator inhibitor-1 (PAI-1) (B) and plasmin antiplasmin (PAP) complexes (C) after infusion of $2 \mathrm{ng} / \mathrm{kg}$ bodyweight and colistin or placebo. Presented are means $\pm 95 \% \mathrm{Cl}$. Colistin significantly reduced plasma concentrations of t-PA $(p<0.001)$, PAI-1 $(p=0.02)$ and PAI-1 $(p<0.001) .{ }^{*} p<0.05 \# p<0.001$ $(n=15)$. period, PAI-1 levels were numerically lower compared to placebo $(\mathrm{p}=0.02,>$ Figure 3$)$.

\section{Endothelial specific biomarkers}

Colistin reduced E-selectin levels compared to placebo 4 h, 6 h, 8 h and $24 \mathrm{~h}$ after the LPS ( $\mathrm{p}=0.001$, $>$ Figure 4$)$. E-Selectin increased two-fold in the colistin period and four-fold in the placebo period compared to baseline. Infusion of colistin also decreased the LPSinduced increase in vWF antigen $(\mathrm{p}=0.001,>$ Figure 4$)$. Colistin almost completely abolished the increase in vWF antigen levels. The maximum increase was measured after $24 \mathrm{~h}$, which was $28 \%$ higher compared to baseline. In contrast, in the placebo period von Willebrand factor increased two-fold $2 \mathrm{~h}$ after infusion of LPS.

\section{Adverse events}

Colistin infusion was well tolerated. Serum creatinine did not increase 24 h post-dose. Following LPS injection, many subjects experienced flu-like symptoms. After colistin infusion, five (33.3\%) cases of shivering, three $(20 \%)$ cases of headache, and four (26.7\%) cases of joint pain occurred. After infusion of placebo 10 subjects $(66.7 \%)$ reported shivering, eight $(53.3 \%)$ reported headache, and five (33.3\%) reported joint pain. Two subjects (13.3\%) experienced a mild syncope in the placebo period.

\section{Discussion}

This placebo controlled trial investigated the actions of colistin on the activation of the coagulation system, the fibrinolytic system and the endothelium in a human endotoxaemia model. The results show that infusion of colistin i) markedly reduces PAI-1, t-PA antigen levels and PAP complexes, ii) reduces soluble E-selectin and vWF, markers of endothelial activation, and iii) slightly delays the onset of the activation of the coagulation system without reducing the magnitude of the activation as assessed by F1+2 levels, TAT complexes or platelet counts.

Colistin binds and neutralises LPS and thereby reduces cytokine levels, such as TNF- $\alpha$, IL- $1 \beta$ or IL- 6 , in animal models $(5,8$, $27,28)$. These findings were recently confirmed in human endotoxaemia: in the same experimental setup infusion of colistin before the infusion of the LPS bolus at $2 \mathrm{ng} / \mathrm{kg}$ bodyweight reduced the LPS-induced increase in TNF- $\alpha \sim 6$-fold, in IL- $6 \sim 4$-fold and in IL-8 3-fold (26). This also translated in a reduced increase in C-reactive protein by $\sim 35 \%$ compared to placebo (26). High-density lipoproteins also bind and neutralise LPS and significantly reduced LPS-induced coagulation activation and fibrinolysis (29). Based on the similar mechanism of action we hypothesised to find similar effects of colistin. However, we only detected a slight delay, but no significant reductions of peak concentrations of $\mathrm{F} 1+2$ or TAT complexes. This is also reflected by the transient decrease in the platelet count, which was similar in both study periods ( $>$ Figure 3). In contrast to that, infusion of colistin almost entirely blunted the activation of the fibrinolytic system. Although colistin 
Figure 4: E-selectin (A) and von Willebrand factor antigen levels (B) after infusion of 2 $\mathrm{ng} / \mathrm{kg}$ bodyweight and colistin or placebo. Presented are means $\pm 95 \% \mathrm{Cl}$. Colistin significantly reduced plasma concentrations of E-selectin $(p=0.001)$ and of von Willebrand factor antigen levels $(p=0.001)$. ${ }^{*} p<0.05 \# p<0.001$ $(n=15)$.

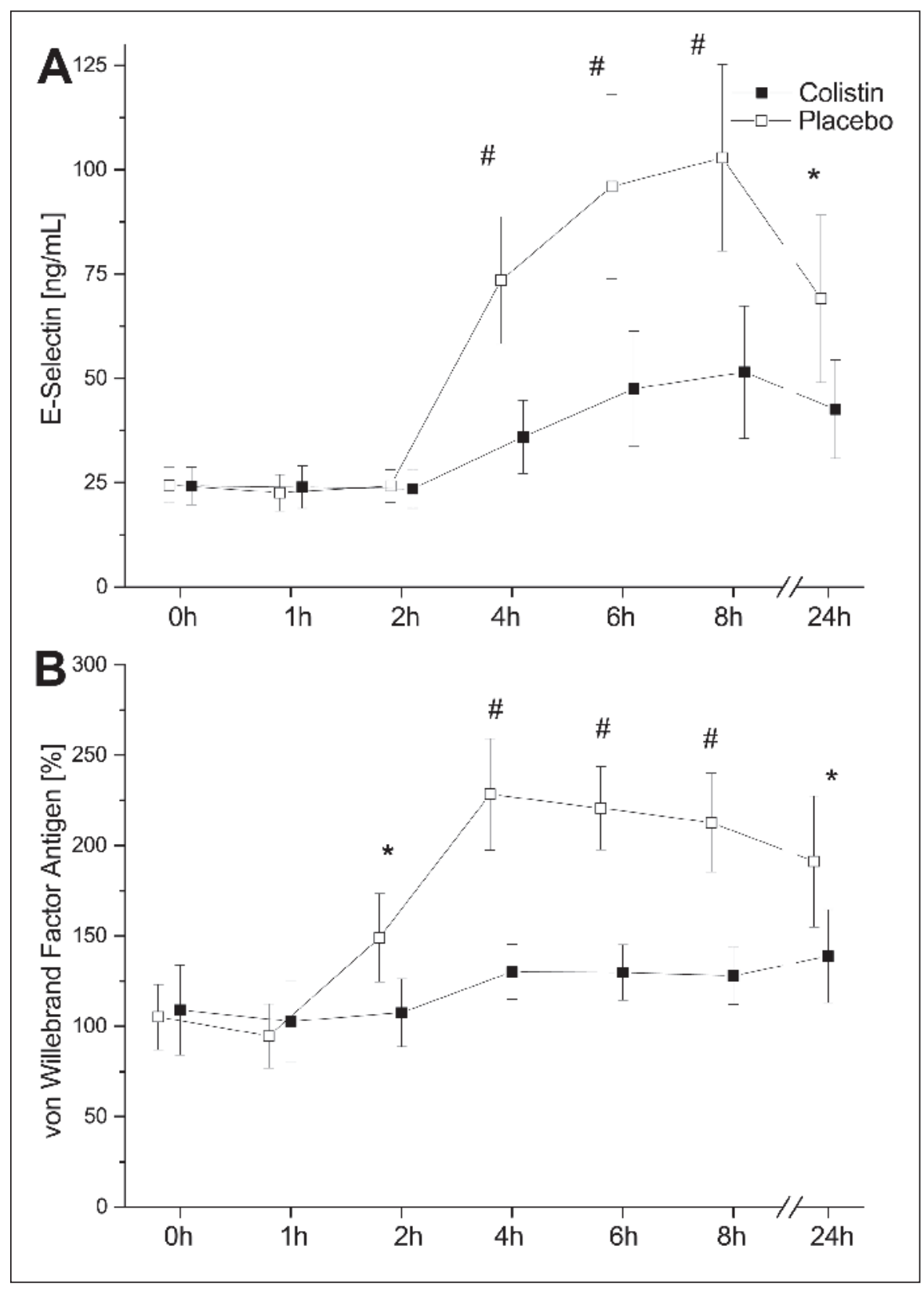

and high-density lipoprotein both bind to the lipid A portion of LPS, they differ in their activity against LPS, potentially due to differences in molecule size and the processing of LPS $(30,31)$.

The current understanding of endotoxaemia involves the LPSbinding protein, which forms a complex by binding to the lipid A portion of LPS. These complexes are recognised by CD14 expressed on monocytes and activate them (32). CD14, together with an accessory protein MD-2, facilitates the activation of toll like receptor 4 , which downstream activates the NF-kB pathway with consecutive cytokine production $(33,34)$. Tissue factor is expressed and possibly transferred to platelets and neutrophils by the formation of microparticles by monocytes, macrophages and endothelial cells (35). The presence of tissue factor subsequently acti- vates the coagulation system via factor VII and finally the conversion of prothrombin to thrombin (36). Interestingly, infusion of an anti-CD14 antibody did not reduce the generation of F1+2, but reduced PAP and tPA concentrations after infusion of an LPS bolus (37).

The activation of the coagulation and the fibrinolytic system are intertwined, but regulated separately during endotoxaemia. Tissue factor drives coagulation activation, while fibrinolysis is mainly mediated by TNF- $a$ (38). Tissue factor pathway inhibitor and active site inhibited factor VIIa dose-dependently inhibited coagulation activation during endotoxaemia without affecting the fibrinolytic or the inflammatory response (39). However, TNF- $\alpha$ may also be involved in the activation of coagulation to a certain 


\section{What is known about this topic?}

- Colistin covalently binds to lipopolysaccharide (LPS) and thereby may exert beneficial effects during endotoxaemia.

- In animal models colistin prevented LPS-induced disseminated intravascular coagulation.

- Anti-inflammatory effects of colistin were shown in human and animal models of endotoxaemia.

\section{What does this paper add?}

- Infusion of colistin blunted the LPS-induced endothelial activation in healthy volunteers.

- Colistin dampened the fibrinolytic response to LPS during endotoxaemia.

- Colistin slightly delayed the activation of coagulation, although its magnitude remained unaffected.

extent. Interestingly, a similar delay in the activation of coagulation was found after blockade of TNF- $\alpha$ during endotoxaemia (16). Thus, we assume that TNF- $\alpha$ is involved in the early phase of coagulation activation, whereas blockade of IL- 6 did not reduce endotoxin-induced activation of coagulation in humans (40). A direct LPS-induced effect on tissue factor bearing cells is likely a driving factor at later stages of endotoxaemia.

The activation of coagulation in inflammatory states is counterbalanced by an activation of the fibrinolytic system. In contrast to the largely unaffected activation of coagulation, the response of the fibrinolytic system was blunted by the infusion of colistin. In the placebo period, LPS markedly increased t-PA, PAI-1 and PAP. The observed changes are in line with previous trials using the human endotoxaemia model (12) and to the well-known changes during acute sepsis (36). As discussed above, these effects are mainly attributable to a reduction in TNF- $a$ levels.

Colistin markedly reduced the LPS induced increase in E-selectin and vWF antigen, most likely due to a reduction in TNF- $\alpha$ (26), because TNF- $\alpha$ inhibitors blunt their release during endotoxaemia $(16,41)$. This endothelium protective effect may prove beneficial for patients suffering from endotoxaemia, as the TNF- $\alpha$ endothelium axis is involved in endothelial barrier disruption, increased vascular permeability and leukocyte transmigration (42). The activation of the endothelium and the consecutive expression of adhesion molecules may be central to the development of sepsis-induced multiple organ failure (43). VWF captures and localises platelets to the vessel wall (44). In sepsis or systemic inflammatory response syndrome endothelial cells release vWF and platelets aggregate, which consecutively causes thrombocytopenia and may lead to consumptive coagulopathy or even a clinical picture resembling thrombotic thrombocytopenic purpura $(45,46)$. Interestingly, E-selectin and vWF antigen correlated with the Simpflied Acute Physiology Score and the Multi Organ Failure Score, which predict mortality, with the severity of systemic inflammatory response syndrome and with mortality $(47,48)$.
One of the major limitations of the trial is that the LPS bolus was injected after infusion of colistin. In patients this seldom may be the case, as antimicrobial treatment usually does not precede infections. LPS is usually quickly bound to transfer proteins, cleared from circulation by the liver and detoxified (49). LPS was detectable for approximately $30 \mathrm{~min}$ after bolus injection (50). Unbound LPS or LPS bound to transfer proteins triggers the inflammatory response (49). We hypothesised that colistin binds and neutralises unbound LPS and thereby reduces its pro-inflammatory actions. Thus, for our model, in order to elucidate colistin's anti-endotoxin effect at its possibly highest magnitude, the presence of colistin in plasma at the time of the LPS injection was necessary.

In certain clinical situations, such as gram-negative gut infections, or abscesses, in times when the gastrointestinal barrier function is impaired and for all infections in which the infectious focus is not immediately resolved by treatment, repetitive bouts of endotoxaemia may occur. In these patients, colistin may provide beneficial effects beside its antimicrobial activity.

Another limitation was that we were only able to detect tissuetype plasminogen activator activity $2 \mathrm{~h}$ after infusion of LPS, because the activity was below the detection limit of the applied assay at all other time-points.

In conclusion, infusion of colistin markedly reduced the endothelial activation, the fibrinolytic response and in human endotoxaemia, while the activation of coagulation remained unaffected. Thus, in patients with gram-negative sepsis colistin may alter the course of the disease, independent of its antimicrobial activity.

\section{Acknowledgements}

The authors would like to thank Edith Lackner, Christa Drucker and Karin Petroczi for their invaluable help throughout the trial.

\section{Conflicts of interest}

None declared.

\section{References}

1. Falagas ME, Kasiakou SK. Colistin: the revival of polymyxins for the management of multidrug-resistant gram-negative bacterial infections. Clin Infect Dis 2005; 40: 1333-1341.

2. Ordooei Javan A, Shokouhi S, Sahraei Z. A review on colistin nephrotoxicity. Eur J Clin Pharmacol 2015; 71: 801-810.

3. Li J, Nation RL, Turnidge JD, et al. Colistin: the re-emerging antibiotic for multidrug-resistant Gram-negative bacterial infections. Lancet Infect Dis 2006; 6: 589-601.

4. Herrmann G, Yang L, Wu H, et al. Colistin-tobramycin combinations are superior to monotherapy concerning the killing of biofilm Pseudomonas aeruginosa. J Infect Dis 2010; 202: 1585-1592.

5. Boisson M, Gregoire N, Couet W, Mimoz O. Colistin in critically ill patients. Minerva Anestesiol 2013; 79: 200-208.

6. Schindler M, Osborn MJ. Interaction of divalent cations and polymyxin B with lipopolysaccharide. Biochemistry 1979; 18: 4425-4430.

7. Koike M, Iida K, Matsuo T. Electron microscopic studies on mode of action of polymyxin. J Bacteriol 1969; 97: 448-452.

8. Aoki N, Tateda K, Kikuchi Y, et al. Efficacy of colistin combination therapy in a mouse model of pneumonia caused by multidrug-resistant Pseudomonas aeruginosa. J Antimicrob Chemother 2009; 63: 534-542. 
9. Cirioni O, Ghiselli R, Orlando F, et al. Efficacy of colistin/rifampin combination in experimental rat models of sepsis due to a multiresistant Pseudomonas aeruginosa strain. Crit Care Med 2007; 35: 1717-1723.

10. Corrigan JJ, Jr., Bell BM. Comparison between the polymyxins and gentamicin in preventing endotoxin-induced intravascular coagulation and leukopenia. Infect Immun 1971; 4: 563-566.

11. Matzneller P, Gobin P, Lackner E, Zeitlinger M. Feasibility of microdialysis for determination of protein binding and target site pharmacokinetics of colistin in vivo. J Clin Pharmacol 2015; 55: 431-437.

12. Mayr FB, Jilma B. Coagulation interventions in experimental human endotoxaemia. Transl Res 2006; 148: 263-271.

13. Pernerstorfer T, Hollenstein U, Hansen J, Knechtelsdorfer M, Stohlawetz P, Graninger W, et al. Heparin blunts endotoxin-induced coagulation activation. Circulation 1999; 100: 2485-2490.

14. Dubor F, Dosne AM, Chedid LA. Effect of polymyxin B and colimycin on induction of plasminogen antiactivator by lipopolysaccharide in human endothelial cell culture. Infect Immun 1986; 52: 725-729.

15. Boos CJ, Mayr FB, Lip GY, Jilma B. Endotoxaemia enhances circulating endothelial cells in humans. J Thromb Haemost 2006; 4: 2509-2511.

16. van der Poll T, Coyle SM, Levi M, et al. Effect of a recombinant dimeric tumor necrosis factor receptor on inflammatory responses to intravenous endotoxin in normal humans. Blood 1997; 89: 3727-3734.

17. Hollenstein UM, Pernerstorfer T, Homoncik M, et al. Effect of factor X inhibition on coagulation activation and cytokine induction in human systemic inflammation. J Infect Dis 2002; 186: 1270-1276.

18. Hollenstein U, Homoncik M, Stohlawetz PJ, et al. Endotoxin down-modulates granulocyte colony-stimulating factor receptor (CD114) on human neutrophils. J Infect Dis 2000; 182: 343-346.

19. Suffredini AF, Fromm RE, Parker MM, et al. The cardiovascular response of normal humans to the administration of endotoxin. N Engl J Med 1989; 321: 280-287.

20. Pernerstorfer T, Schmid R, Bieglmayer C, et al. Acetaminophen has greater antipyretic efficacy than aspirin in endotoxaemia: a randomized, double-blind, placebo-controlled trial. Clin Pharmacol Ther 1999; 66: 51-57.

21. Ruzicka K, Veitl M, Thalhammer-Scherrer R, Schwarzinger I. The new hematology analyzer Sysmex XE-2100: performance evaluation of a novel white blood cell differential technology. Arch Pathol Lab Med 2001; 125: 391-396.

22. Jilma-Stohlawetz P, Gilbert JC, Gorczyca ME, et al. A dose ranging phase I/II trial of the von Willebrand factor inhibiting aptamer ARC1779 in patients with congenital thrombotic thrombocytopenic purpura. Thromb Haemost 2011; 106: 539-547.

23. Derhaschnig U, Pernerstorfer T, Knechtelsdorfer M, et al. Evaluation of antiinflammatory and antiadhesive effects of heparins in human endotoxaemia. Crit Care Med 2003; 31: 1108-1112.

24. Spiel AO, Derhaschnig U, Schwameis M, et al. Effects of prasugrel on platelet inhibition during systemic endotoxaemia: a randomized controlled trial. Clin Sci 2012; 123: 591-600.

25. Mayr FB, Spiel AO, Leitner JM, et al. Racial differences in endotoxin-induced tissue factor-triggered coagulation. J Thromb Haemost 2009; 7: 634-640.

26. Matzneller P, Strommer S, Drucker C, et al. Colistin reduces LPS-triggered inflammation in a human sepsis model in vivo: A randomized controlled trial. Clin Pharmacol Ther 2016; Epub ahead of print.

27. Senturk S. Evaluation of the anti-endotoxic effects of polymyxin-E (colistin) in dogs with naturally occurred endotoxic shock. J Vet Pharmacol Ther 2005; 28: 57-63.

28. Warren HS, Kania SA, Siber GR. Binding and neutralisation of bacterial lipopolysaccharide by colistin nonapeptide. Antimicrob Agents Chemother 1985; 28: 107-112.

29. Pajkrt D, Lerch PG, van der Poll T, et al. Differential effects of reconstituted high-density lipoprotein on coagulation, fibrinolysis and platelet activation during human endotoxaemia. Thromb Haemost 1997; 77: 303-307.
30. Van Lenten BJ, Fogelman AM, Haberland ME, Edwards PA. The role of lipoproteins and receptor-mediated endocytosis in the transport of bacterial lipopolysaccharide. Proc Natl Acad Sci USA 1986; 83: 2704-2708.

31. Soon RL, Velkov T, Chiu F, et al. Design, synthesis, and evaluation of a new fluorescent probe for measuring polymyxin-lipopolysaccharide binding interactions. Anal Biochem 2011; 409: 273-283.

32. Thaler B, Hohensinner PJ, Krychtiuk KA, et al. Differential in vivo activation of monocyte subsets during low-grade inflammation through experimental endotoxaemia in humans. Sci Rep 2016; 6: 30162.

33. Shimazu R, Akashi S, Ogata H, et al. MD-2, a molecule that confers lipopolysaccharide responsiveness on Toll-like receptor 4. J Exp Med 1999; 189: 1777-1782.

34. Lorne E, Dupont H, Abraham E. Toll-like receptors 2 and 4: initiators of nonseptic inflammation in critical care medicine? Intensive Care Med 2010; 36: 1826-1835.

35. Mooberry MJ, Bradford R, Hobl EL, et al. Procoagulant microparticles promote coagulation in a factor XI-dependent manner in human endotoxaemia. J Thromb Haemost 2016; 14: 1031-1042.

36. Semeraro N, Ammollo CT, Semeraro F, Colucci M. Coagulopathy of Acute Sepsis. Semin Thromb Hemost 2015; 41: 650-658.

37. Verbon A, Meijers JC, Spek CA, et al. Effects of IC14, an anti-CD14 antibody, on coagulation and fibrinolysis during low-grade endotoxaemia in humans. J Infect Dis 2003; 187: 55-61.

38. van Hinsbergh VW, Bauer KA, Kooistra T, et al. Progress of fibrinolysis during tumor necrosis factor infusions in humans. Concomitant increase in tissue-type plasminogen activator, plasminogen activator inhibitor type-1, and fibrin(ogen) degradation products. Blood 1990; 76: 2284-2289.

39. de Jonge E, Dekkers PE, Creasey AA, et al. Tissue factor pathway inhibitor dosedependently inhibits coagulation activation without influencing the fibrinolytic and cytokine response during human endotoxaemia. Blood 2000; 95: 1124-1129.

40. Derhaschnig U, Bergmair D, Marsik C, et al. Effect of interleukin-6 blockade on tissue factor-induced coagulation in human endotoxaemia. Crit Care Med 2004; 32: $1136-1140$.

41. DeLa Cadena RA, Majluf-Cruz A, Stadnicki A, et al. Recombinant tumor necrosis factor receptor p75 fusion protein (TNFR: Fc) alters endotoxin-induced activation of the kinin, fibrinolytic, and coagulation systems in normal humans. Thromb Haemost 1998; 80: 114-118.

42. Laviola L, Orlando MR, Incalza MA, et al. TNFalpha signals via p66(Shc) to induce E-selectin, promote leukocyte transmigration and enhance permeability in human endothelial cells. PLoS One 2013; 8: e81930.

43. Reinhart K, Bayer O, Brunkhorst F, Meisner M. Markers of endothelial damage in organ dysfunction and sepsis. Crit Care Med 2002; 30 (5 Suppl): S302-312.

44. Methia N, Andre P, Denis CV, et al. Localized reduction of atherosclerosis in von Willebrand factor-deficient mice. Blood 2001; 98: 1424-1428.

45. Akca S, Haji-Michael P, de Mendonca A, et al. Time course of platelet counts in critically ill patients. Crit Care Med 2002; 30: 753-756.

46. Schwameis M, Schorgenhofer C, Assinger A, et al. VWF excess and ADAMTS13 deficiency: a unifying pathomechanism linking inflammation to thrombosis in DIC, malaria, and TTP. Thromb Haemost 2015; 113: 708-718.

47. Kayal S, Jais JP, Aguini N, et al. Elevated circulating E-selectin, intercellular adhesion molecule 1, and von Willebrand factor in patients with severe infection. Am J Respir Crit Care Med 1998; 157: 776-784.

48. Iba T, Gando S, Murata A, et al. Predicting the severity of systemic inflammatory response syndrome (SIRS)-associated coagulopathy with hemostatic molecular markers and vascular endothelial injury markers. J Trauma 2007; 63: 1093-1098.

49. Walley KR, Thain KR, Russell JA, et al. PCSK9 is a critical regulator of the innate immune response and septic shock outcome. Sci Transl Med 2014; 6: 258ra143.

50. van Deventer SJ, Buller HR, ten Cate JW, et al. Experimental endotoxaemia in humans: analysis of cytokine release and coagulation, fibrinolytic, and complement pathways. Blood 1990; 76: 2520-2526. 